\title{
Impact of Frailty on the Risk of Exacerbations and All-Cause Mortality in Elderly Patients with Stable Chronic Obstructive Pulmonary Disease
}

This article was published in the following Dove Press journal:

Clinical Interventions in Aging

Jia Luo

Dai Zhang (D)

Wen Tang

Li-Yang Dou

Ying Sun

Department of Geriatrics, Beijing Friendship Hospital, Capital Medical University, Beijing, 100050, People's Republic of China
Correspondence: Ying Sun Department of Geriatrics, Beijing

Friendship Hospital, Capital Medical

University, No. 95, Yong'an Road, Xicheng

District, Beijing, I00050, People's

Republic of China

$\mathrm{Tel}+86 \quad 1063137737$

$\mathrm{Fax}+861063138795$

Emailysun15@I63.com

Dai Zhang

Department of Geriatrics, Beijing

Friendship Hospital, Capital Medical

University, Beijing, I00050, People's

Republic of China

Email zhangdai0806@I26.com
Rationale: For the high prevalence of frail in patients with chronic obstructive pulmonary disease (COPD), further study should explore an in-depth understanding of the relationship between frailty and prognosis of COPD.

Objective: To determine the correlation between frailty and risk of acute exacerbation, hospitalizations, and mortality in older patients with stable COPD.

Participants and Methods: Consecutive older adults $(\geq 65)$ diagnosed with stable COPD from January 2018 to July 2019, with an average follow-up of 546 days $(\mathrm{N}=309)$. Frailty was defined by the Fried frailty phenotype. Poisson regression was performed to assess the influence of frailty on the incidence of acute exacerbations of COPD (AECOPD) and allcause hospitalizations in a year. Cox regression was performed to evaluate the effect of frailty on all-cause mortality in patients with stable COPD.

Results: The prevalence of frailty was $49.8 \%$. The most common phenotypic characteristics were weakness $(99.4 \%)$ followed by slowness $(92.9 \%)$. After adjustment, frailty increased the incidence of AECOPD (IRR $=1.75,95 \% \mathrm{CI}: 1.09-2.82$ ) and all-cause hospitalizations $($ IRR $=1.39,95 \%$ CI 1.04-1.87) within a year. Slowness was associated with AECOPD (IRR $=1.77,95 \%$ CI: $1.03-3.03$ ), and weakness was associated with increased all-cause hospitalizations (IRR $=1.53,95 \%$ CI: 1.04-2.25). The all-cause mortality risk was more than twofold higher in frail patients $(\mathrm{HR}=2.54,95 \% \mathrm{CI}: 1.01-6.36)$ than non-frail patients. Low physical activity $(\mathrm{HR}=2.66,95 \% \mathrm{CI}: 1.17-6.05)$ and weight loss $(\mathrm{HR}=2.15,95 \% \mathrm{CI}$ : $1.02-4.51)$ were significantly associated with increased all-cause mortality in patients with COPD.

Conclusion: Frailty increased the incidence of acute exacerbation and hospitalization, as well as increased mortality in older patients with stable COPD. This knowledge will help physicians identify high-risk groups with COPD and frailty who may benefit from targeted interventions to prevent disease progression.

Keywords: COPD, frailty, older adults, acute exacerbation, mortality

\section{Introduction}

Chronic obstructive pulmonary disease (COPD) is a common preventable and treatable disease characterized by persistent respiratory symptoms and airflow limitation. COPD is related to exposure of the body to toxic gases or toxic particles, causing abnormalities in the respiratory tract and/or alveoli. ${ }^{1}$ In the past three decades, the incidence and mortality of COPD in China have declined significantly. However, with continuous exposure to environmental risk factors and with an aging 
population, COPD remains the fourth leading cause of death in China. ${ }^{2}$ COPD is considered a disease that accelerates aging, and it is related to the accumulation of multiple impairments in pulmonary function during the aging process. Frailty is a geriatric syndrome that is defined as a cumulative decline in multiple organ systems and loss of physiological reserves, increasing the vulnerability to adverse outcomes, including falls, delirium, disability, reduced quality of life, hospitalization, and mortality in elderly adults. ${ }^{3}$ The Fried phenotype is well established and widely used for evaluating frailty. It evaluates five aspects including nutritional status, physical activity, mobility, strength, and energy. ${ }^{4}$ Frailty may be prevalent in as many as half of patients with COPD, especially in elderly patients, is strongly related to airflow limitation, symptoms, and exacerbation frequency, and can be mitigated through pulmonary rehabilitation. ${ }^{5-10}$

COPD is a heterogeneous disease. Even patients with the same forced expiratory volume in 1 second $\left(\mathrm{FEV}_{1}\right)$ have considerable differences in symptoms, exacerbations, response to therapy, the rate of disease progression, or death, therefore they exhibit different clinical phenotypes. ${ }^{11}$ Phenotypic recognition not only helps in understanding this heterogeneity, but it also has potential diagnostic and therapeutic importance. The impact of frailty on a person's meaningful outcomes can identify a unique cluster of patients. Physical frailty traits, although not well defined in patients with COPD, have long been recognized as having direct and independent therapeutic implications for management, in addition to substantially impacting quality of life, prognosis, and health care utilization. ${ }^{12}$

Previous researches in COPD and frailty were mainly cross-sectional or retrospective studies, but not longitudinal studies. In addition, the frailty assessment tools used in these studies were various. The correlation between phenotypic characteristics of frailty and prognosis of COPD has not been fully understood. In this study, we aimed to assess the association between frailty and COPD adverse outcomes and to further evaluate the correlation between different phenotype characteristics of frailty and COPD prognosis. We hypothesized that frailty could independently predict the mortality or exacerbation of elderly patients with stable COPD. This information may help in early identification of high-risk patients, hierarchical management of interventional factors, and prognostic improvement of elderly patients with stable COPD.

\section{Methods}

\section{Participants}

This was an observational study of prospectively collected data from the Beijing Friendship Hospital, a tertiary hospital affiliated with Capital Medical University, China. We enrolled all consecutive older patients with a diagnosis of stable COPD via the geriatric department between January 2018 to July 2019. The inclusion criteria of the study were patients (1) age $\geq 65$ years old; (2) with a diagnosis of COPD based on the 2017 Global Initiative for COPD (GOLD) guidelines, ${ }^{1}$ with respiratory symptoms or risk factors, and a post-bronchodilator ratio of FEV1 to forced vital capacity $(\mathrm{FVC})<0.70$; and (3) with good mental and cognitive status in able to complete frail assessment.

Exclusion criteria of this study were patients (1) with acute exacerbation in the previous 3 months that required a change in medication; (2) with respiratory diseases other than COPD, ie, asthma, active tuberculosis, or diffuse parenchymal lung disease; (3) complicated with unstable cardiac disease or a predominant neurological disability; and (4) with terminal-stage malignant tumor.

\section{Measurements Data Collection}

Trained evaluators performed face-to-face interviews to collect patients' information including demographic characteristics, smoking history, body mass index (BMI), medication, respiratory symptoms, frequencies of acute exacerbations of COPD (AECOPD) and hospitalizations within 1 year.

Spirometry was performed by trained paramedical personnel according to the American Thoracic Society and European Respiratory Society guidelines and the accuracy of the lung function equipment was frequently verified. GOLD severity was classified as mild-, moderate-, severe- or very severeCOPD by forced expiratory volume in $\mathrm{FEV}_{1} \%$ pred of $\geq 80 \%, 50-80 \%, 30-50 \%$, and $<30 \%$, respectively. COPD subjects were also classified A to D group according to the recent GOLD update which includes the evaluation of selfreported symptoms (the COPD Assessment Test [CAT] and modified Medical Research Council [mMRC] dyspnea scale), and the history of moderate (requiring steroids and/or antibiotics), and severe (requiring hospitalization) exacerbations as well as the severity of airflow limitation. A was the mildest symptoms and lowest risk and D was the most severe symptom and highest risk. 


\section{Frailty}

Frailty was assessed using the Fried frailty phenotype (FFP) criteria: ${ }^{4}$ (1) unintentional weight loss $(4.5 \mathrm{~kg}$ in the past 12 months); (2) self-reported exhaustion; (3) weakness; (4) slowness; and (5) low level of physical activity. The cutoff values were consistent with the original criteria. Each construct was considered present (score of 1) or absent (score of 0 ). The total score ranged from 0 to 5 . As established previously, the presence and absence of frailty were defined as a score of $\geq 3$ and $\leq 2$, respectively.

\section{Covariates}

The Charlson comorbidity index (CCI) was performed to assess the burden of complications in patients. ${ }^{13}$ The CCI is the sum of weighted measures of the following 17 chronic diseases: ischemic heart disease, congestive heart failure, cerebrovascular accident, peripheral vascular disease, chronic lung diseases, diabetes, dementia, connective tissue diseases, renal diseases, liver diseases, hemiplegia, solid tumors or hematological malignancies, and acquired immunodeficiency diseases. Each disease has a score of 1, 2,3 , or 6 , depending on the severity.

The nutritional status of patients was evaluated using the Mini Nutritional Assessment-Short Form. ${ }^{14}$ These questionnaire items include BMI, weight loss in the past 3 months, dietary changes, stress or acute illness, degree of mobility, and neuropsychiatric diseases. The total score of the questionnaire is 14 points, and scores from 0 to 7 indicate malnutrition. Functional status was assessed using the Katz Activities of Daily Living (ADL) scale and Lawton Instrumental Activities of Daily Living (IADL) scale. ${ }^{15}$

\section{Follow-Up and Study Outcomes}

Research practitioners phoned participants every three months to collect data on the exacerbation frequencies and hospitalizations. All patients followed up until July 2020. Deaths were confirmed through surveillance or matched to official death records. The primary outcome was all-cause mortality during follow-up. The secondary outcomes were the frequencies of AECOPD and the frequencies of all-cause hospitalizations in a year.

\section{Statistical Analysis}

The normality of continuous variables was tested by the Shapiro-Wilk test. Based on its result, continuous variables were expressed as the means \pm standard deviation (SD) or median (interquartile range $[\mathrm{IQR}] /$ minimum value - maximum value) and compared using Student's $t$-test or the
Mann-Whitney $U$-test as appropriate. Categorical variables were compared using the chi-squared test or Fisher's exact test. The associations between baseline frailty status and moderate-to-severe exacerbations and all-cause hospitalizations were analyzed using Poisson regression. The association between baseline frailty status and all-cause mortality was analyzed using a Log rank test and the Cox proportional hazards model. The following variables were considered as confounding factors and adjusted in the two regression equations: age, gender, CCI, medication, GOLD severity, moderate-to-severe exacerbation history, and CAT score. For patients whose effective follow-up time was less than 1 year, the logarithm of the follow-up time (years) was used as the offset in Poisson regression. All statistical analyses were performed using $\mathrm{R}$ version 3.6.3 (The R Project for Statistical Computing, Vienna, Austria). $P<00.05$ was considered statistically significant.

\section{Results}

\section{Baseline Characteristics of Participants}

This cohort study finally included 309 participants (22\% women), with a median age of 86 years (IQR: $80-90$ years). Demographic characteristics were presented in Table 1. A total of 154/309 (49.8\%) patients were frail. Frail patients were significantly older, had more comorbidity, poorer lung function ( $\mathrm{FEV}_{1} \%$ predicted), more symptoms (CAT or mMRC), and worse history of acute exacerbations or hospitalizations compared to non-frail patients. However, frail patients and comparators were similar in gender, smoking index, and GOLD severity category. Frail patients also had significantly poorer nutritional status, $\mathrm{ADL}$, gait speed, and handgrip strength compared to the non-frail group. Among frail patients, the most common phenotypic characteristics were weakness $(99.4 \%)$ followed by slowness $(92.9 \%)$, exhaustion $(79.9 \%)$, low physical activity (76.6\%), and weight loss (20.1\%) (Figure 1).

\section{Effect of Frailty on AECOPD and All-Cause Hospitalization}

All patients completed follow-up, with an average followup time of 546 days (IQR: 383-733 days). The incidence of acute exacerbations and all-cause hospitalizations in the frail group within a year were significantly higher than those in the non-frail group $(P<0.001$, Table 2$)$. The results of Poisson regression are presented in Table 3. After adjusting for age, gender, medication, CCI, GOLD severity, moderate-to-severe exacerbation history, and 
Table I Baseline Characteristics of Elderly Patients with Stable Chronic Obstructive Pulmonary Disease (COPD), Classified According to Frailty

\begin{tabular}{|c|c|c|c|c|}
\hline & Total Cohort & Non-Frail & Frail & $P$-value \\
\hline $\mathrm{N}(\%)$ & 309 & $155(50.2)$ & $154(49.8)$ & \\
\hline Age, yr, median (IQR) & $86(80,90)$ & $82(77,88)$ & $89(85,92)$ & $<0.00 I^{*}$ \\
\hline Sex, female $n(\%)$ & $68(22.0)$ & $29(18.7)$ & $39(25.3)$ & 0.205 \\
\hline Smoking Status, n (\%) & & & & $0.031^{*}$ \\
\hline Never & $143(46.3)$ & $68(43.9)$ & $75(48.7)$ & \\
\hline Previous & $99(32.0)$ & $44(28.4)$ & $55(35.7)$ & \\
\hline Current & $67(21.7)$ & $43(27.7)$ & $24(15.6)$ & \\
\hline Smoking Index (pack yr), median (IQR) & $5(0,30)$ & $10(0,30)$ & $3(0,30)$ & 0.942 \\
\hline BMI $\left(\mathrm{kg} / \mathrm{m}^{2}\right)$, mean $(\mathrm{SD})$ & 24.I (3.8) & $25.1(3.2)$ & $23.2(4.1)$ & $<0.00 I^{*}$ \\
\hline $\mathrm{CCl}$, median (IQR) & $4(3,5)$ & $3(2,4)$ & $5(3,6)$ & $<0.001^{*}$ \\
\hline MNA-SF, median (IQR) & $12(9,14)$ & $13(12,14)$ & $10(8,12)$ & $<0.00 I^{*}$ \\
\hline Malnutrition, n (\%) & $32(10.5)$ & $6(3.9)$ & $26(17.1)$ & $<0.00 I^{*}$ \\
\hline \multicolumn{5}{|l|}{ COPD Disease Severity } \\
\hline $\mathrm{FEV}, \%$ predicted, median (IQR) & $74.2(60.4,85.3)$ & $76.3(63.8,87.5)$ & $71.0(57.0,83.4)$ & $0.028 *$ \\
\hline GOLD severity category, n (\%) & & & & 0.090 \\
\hline Mild ( $\geq 80 \%)$ & 114 (36.9) & $63(40.6)$ & $51(33.1)$ & \\
\hline Moderate $(50-80 \%)$ & $162(52.4)$ & $81(52.3)$ & $81(52.6)$ & \\
\hline Severe $(30-49 \%)$ & $30(9.7)$ & II (7.I) & $19(12.3)$ & \\
\hline Very severe $(<30 \%)$ & $3(1.0)$ & $0(0)$ & $3(1.9)$ & \\
\hline mMRC, median (IQR) & $2.0(1.0,3.0)$ & $1.0(1.0,2.0)$ & $2.0(2.0,3.0)$ & $<0.00 I^{*}$ \\
\hline $\mathrm{mMRC} \geq 2, \mathrm{n}(\%)$ & $85(27.5)$ & $9(5.8)$ & $76(49.4)$ & $<0.00 I^{*}$ \\
\hline CAT, median (IQR) & $8(3,13)$ & $5(2,9)$ & $12(6,15)$ & $<0.001^{*}$ \\
\hline CAT $\geq 10, \mathrm{n}(\%)$ & $|3|(42.4)$ & $36(23.2)$ & $95(61.7)$ & $<0.00 I^{*}$ \\
\hline$\geq$ I COPD moderate-to-severe exacerbations prior yr, n (\%) & $95(30.7)$ & $26(16.8)$ & $69(44.8)$ & $<0.00 I^{*}$ \\
\hline GOLD stage & & & & $<0.001^{*}$ \\
\hline GOLD A, n (\%) & $129(4 \mid .7)$ & $107(69.0)$ & $22(14.3)$ & \\
\hline GOLD B, n (\%) & $132(42.7)$ & $40(25.8)$ & $92(59.7)$ & \\
\hline GOLD C, n (\%) & $7(2.3)$ & $2(1.3)$ & $5(3.2)$ & \\
\hline GOLD D, n (\%) & $41(13.3)$ & $6(3.9)$ & $35(22.7)$ & \\
\hline ADL, median (IQR) & $5(3,6)$ & $6(5,6)$ & $3(2,5)$ & $<0.00 I^{*}$ \\
\hline IADL, median (IQR) & $5(2,8)$ & $8(6,8)$ & $3(1,5)$ & $<0.00 I^{*}$ \\
\hline \multicolumn{5}{|l|}{ Physical Function } \\
\hline Pace, m/s, median (IQR) & $0.57(0.27,0.88)$ & $0.88(0.67,1.09)$ & $0.30(0,0.45)$ & $<0.00 I^{*}$ \\
\hline Handgrip strength, kg, mean (SD) & $20(13,26)$ & $25(20,32)$ & $14(8,20)$ & $<0.00 I^{*}$ \\
\hline Medication & & & & $0.009 *$ \\
\hline LAMA/LABA, n (\%) & $127(4 \mid . I)$ & $66(42.9)$ & $61(39.4)$ & \\
\hline 2BD/BD+ICS, n (\%) & $24(7.8)$ & $6(3.9)$ & $18(11.6)$ & \\
\hline LAMA+LABA+ICS, n (\%) & $43(13.9)$ & $16(10.4)$ & $27(17.4)$ & \\
\hline None, n (\%) & $115(37.2)$ & $66(42.9)$ & $49(31.6)$ & \\
\hline
\end{tabular}

Notes: ${ }^{\dagger}$ Frailty was defined as Fried phenotype scores $\geq 3$. $* P<0.05$.

Abbreviations: SD, standard deviation; IQR, inter-quartile range; BMI, body mass index; ADL, activity of daily living; IADL, instrumental activity of daily living; CCI, Charlson's comorbidity index; MNA-SF, Mini Nutritional Assessment-Short Form; FEV , forced expiratory volume in I second; GOLD, Global Initiative for COPD; mMRC, modified Medical Research Council; CAT, COPD Assessment Test; BD, bronchodilator; ICS, inhaled corticosteroids; LABA, long-acting beta-agonists; LAMA, long-acting muscarinic antagonists. 


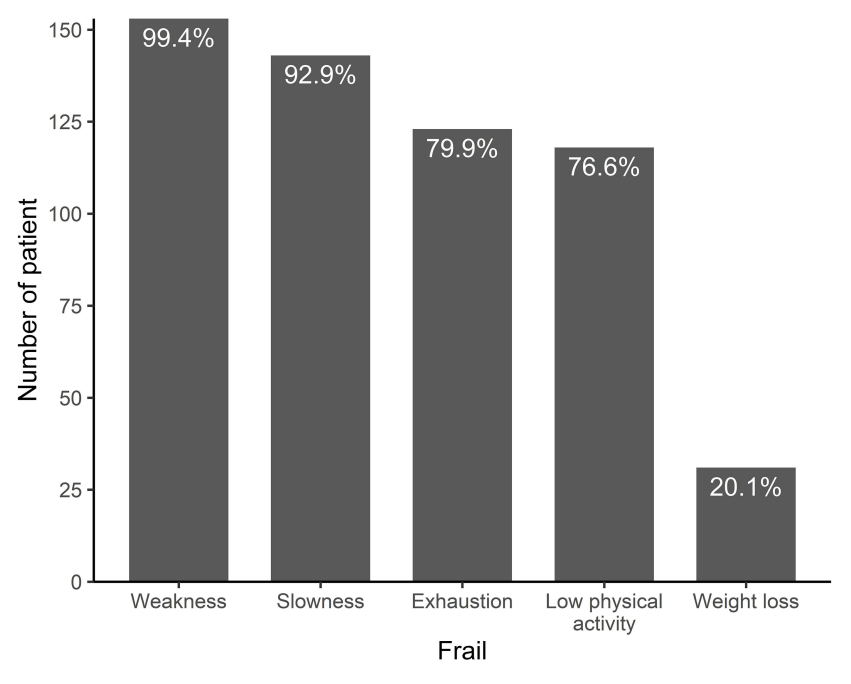

Figure I Distribution of frailty phenotypic characteristics assessed using Fried frailty phenotypes in older patients with stable chronic obstructive pulmonary disease (COPD)

CAT, frailty significantly increased incidence of AECOPD (incidence rate ratio $[\mathrm{IRR}]=1.75,95 \%$ confidence interval [CI]: $1.09-2.82, P=0.021)$ and all-cause hospitalizations $(\mathrm{IRR}=1.39,95 \%$ CI $1.04-1.87, P=0.028)$ within a year. The five phenotypic characteristics in multivariate Poisson regression analysis showed that slowness was associated with AECOPD (IRR $=1.77,95 \%$ CI: 1.03-3.03), and weakness was associated with all-cause hospitalization $(\mathrm{IRR}=1.53,95 \% \mathrm{CI}: 1.04-2.25)$.

\section{Effect of Frailty on All-Cause Mortality in Patients with COPD}

As shown in Table 2, there were 7 deaths (4.5\%) in the non-frail group and 43 deaths $(27.9 \%)$ in the frail group during the follow-up period. Frail participants had significantly worse survival by Kaplan-Meier survival analysis (see Figure 2A). After adjustment, Cox regression analysis showed that the all-cause mortality risk was more than twofold higher in frail patients (hazard ratio $[\mathrm{HR}]=2.54$, 95\% CI: $1.01-6.36, P=0.047)$ than non-frail patients (Figure 2B). Low physical activity $(\mathrm{HR}=2.66,95 \% \mathrm{CI}$ : 1.17-6.05) and weight loss $(\mathrm{HR}=2.15$, 95\% CI: $1.02-$ 4.51) were significantly associated with increased allcause mortality in patients with COPD. (Table 3)

\section{Discussion}

This study demonstrated that frailty was prevalent in stable COPD elderly population. Frail COPD patients had a $75 \%$ increased risk of AECOPD within a year, and a $39 \%$ higher risk of all-cause hospitalization. The risk of allcause mortality in the frail group increased significantly by $154 \%$ compared with the non-frail group during followup. For the five phenotypic characteristics of frailty, low physical activity and weight loss were significantly associated with increased mortality in elderly patients with COPD.

Maddocks et $\mathrm{al}^{8}$ stated that the prevalence of frailty in elderly patients with COPD was significantly higher than that of patients without COPD (10.2\% vs 3.4\%). However, owing to the heterogeneity of the study population and frailty assessment tools, the prevalence of frailty in COPD varies significantly, ranging from $6.6 \%$ to $75.5 \% .^{5,8-10}$ In this study, we performed the FFP assessment to evaluate very old patients with stable COPD, showing a prevalence of frailty of $49.8 \%$, which was higher compared to other studies. $^{5,10,16,17}$ In our cohort, frail patients presented worse nutritional status and physical performance compared to the non-frail group. Weakness (99.4\%) and slowness $(92.9 \%)$ were the most common phenotypic

Table 2 Results for I-Year Acute Exacerbation of COPD, All-Cause Hospitalization, and Mortality in Frail and Non-Frail Elderly Patients with Stable COPD

\begin{tabular}{|c|c|c|c|c|c|}
\hline Outcome & $\begin{array}{l}\text { Total Cohort } \\
\qquad(n=309)\end{array}$ & $\begin{array}{l}\text { Non-Frail }(n= \\
\text { 155) }\end{array}$ & $\begin{array}{c}\text { Frail }^{\dagger} \\
(n=I 54)\end{array}$ & $\begin{array}{l}\text { Crude IRR/HR } \\
\qquad(95 \% \mathrm{Cl})\end{array}$ & $P$-value \\
\hline $\begin{array}{l}\text { Moderate-to-severe exacerbations per year, median } \\
(\min , \max )\end{array}$ & $0(0,5)$ & $0(0,2)$ & $0(0,5)$ & $4.48(3.03-6.85)$ & $<0.00 I^{*}$ \\
\hline $\begin{array}{l}\text { All-cause hospitalizations per year, median (min, } \\
\max \text { ) }\end{array}$ & $I(0,6)$ & $0(0,3)$ & $I(0,6)$ & $2.45(1.92-3.12)$ & $<0.00 I^{*}$ \\
\hline All-cause I-year death, n (\%) & $28(9.1)$ & $3(1.9)$ & $25(16.2)$ & $9.00(2.72,29.83)$ & $<0.00 I^{*}$ \\
\hline All-cause death during follow-up, $n$ (\%) & $50(16.2)$ & $7(4.5)$ & $43(27.9)$ & $6.94(3.12,15.42)$ & $<0.00 I^{*}$ \\
\hline
\end{tabular}

Notes: ${ }^{\dagger}$ Frailty was defined as Fried phenotype scores $\geq 3$. $* P<0.05$.

Abbreviations: IRR, incidence rate ratio; HR, hazard ratios; $\mathrm{Cl}$, confidence intervals. 
Table 3 Effects of Frailty and Its Characteristics on I-Year Acute Exacerbation, All-Cause Hospitalization, and All-Cause Mortality in Older Patients with Stable COPD

\begin{tabular}{|c|c|c|c|c|c|c|}
\hline & \multicolumn{2}{|c|}{ Moderate-Severe COPD Exacerbations } & \multicolumn{2}{|c|}{ All-Cause Hospitalizations } & \multicolumn{2}{|c|}{ All-Cause Mortality } \\
\hline & IRR (95\% CI) & $P$-value & IRR (95\% CI) & $P$-value & HR (95\% Cl) & $P$-value \\
\hline Non-frail & ref & & ref & & ref & \\
\hline Frail $(\geq 3)^{\dagger}$ & $1.75(1.09-2.82)$ & $0.021 *$ & $1.39(1.04-1.87)$ & $0.028^{*}$ & $2.54(1.01-6.36)$ & $0.047^{*}$ \\
\hline \multicolumn{7}{|c|}{ Individual frailty characteristics } \\
\hline Exhaustion & $1.29(0.88-1.89)$ & 0.193 & I.II (0.87-I.43) & $0.40 \mathrm{I}$ & $1.33(0.66-2.66)$ & 0.418 \\
\hline Weakness & $1.92(0.99-3.73)$ & 0.055 & $1.53(1.04-2.25)$ & $0.029 *$ & $6.39(0.77-52.98)$ & 0.084 \\
\hline Slowness & $1.77(1.03-3.03)$ & $0.038^{*}$ & $1.17(0.85-1.59)$ & 0.334 & $2.11(0.74-6.02)$ & 0.156 \\
\hline Low physical activity & $1.35(0.88-2.09)$ & 0.172 & $1.00(0.76-1.32)$ & 0.996 & $2.66(1.17-6.05)$ & $0.02 I^{*}$ \\
\hline Weight loss & I.07 (0.68-I.70) & 0.768 & $0.96(0.67-1.35)$ & 0.798 & $2.15(I .02-4.5 I)$ & $0.044^{*}$ \\
\hline
\end{tabular}

Notes: Data were estimated incidence rate ratios and $95 \%$ confidence intervals of the explanatory variables in the I-year acute exacerbation and all-cause hospitalization, and hazard ratios and $95 \%$ confidence intervals of the explanatory variables in the all-cause mortality. IRR and HR were both adjusted for age, gender, CCl, medication, GOLD severity, moderate-to-severe exacerbation history, and CAT. ${ }^{\dagger}$ Frailty was defined as Fried Phenotype scores $\geq 3 . * P<0.05$.

Abbreviations: IRR, incidence rate ratio; $\mathrm{HR}$, hazard ratios; $\mathrm{Cl}$, confidence intervals.

characteristics of the frail group. Sarcopenia is a syndrome characterized by low levels of muscle strength, muscle quantity/quality, and physical performance is an indicator of its severity. ${ }^{18-20}$ The physical phenotype of frailty shows significant overlap with sarcopenia, low handgrip strength (weakness) and slow gait speed (slowness) are characteristic of both. ${ }^{21,22}$ Measurement of weakness and slowness can reflect the muscle strength and physical fitness of patients. This method of evaluation is rapid, simple, economical, and practical. Non-fat weight loss affects the function of respiratory muscles and peripheral muscles in patients with COPD, manifesting by decreased muscle strength, exercise intolerance, and fatigue. Skeletal muscle atrophy and muscle weakness in patients with COPD are more prominent in the lower limbs than in the upper limbs, while the reduced quadriceps strength is mostly a reflection of the loss in muscle mass. ${ }^{23-25}$ Patients with COPD often have dyspnea, muscle atrophy, and exercise intolerance, creating a vicious cycle. For example, impaired lung function limits the exercise ability of patients with COPD, while long-term reduced activity level leads to skeletal muscle disuse atrophy and exercise intolerance, further exacerbating dyspnea, manifested by gradually limited activity. ${ }^{26,27}$ Therefore, it is not surprising that frailty is prevalent in patients with COPD.

Bernabeu-Mora et al confirmed that frailty predicted the risk of early hospital readmission for inpatients with AECOPD, adjusted for age, comorbidities, and other confounding factors, frailty increased 5.19-fold risk for 90day readmission in patients with COPD (odds ratio $=5.19$;
95\% CI: $1.26-21.50) .{ }^{28}$ Our study demonstrated that frailty increased the risk of AECOPD (IRR $=1.75,95 \%$ CI: $1.09-2.82)$ and all-cause hospitalization (IRR $=1.39$, 95\% CI 1.04-1.87) within a year in elderly patients with stable COPD, slowness and weakness were the outstanding phenotypic characteristics. However, it is necessary to point out that with the accumulation of various physiological deficits, frailty affected the clinical outcomes of patients with COPD, which is more in line with the actual clinical situation. Frailty not only increases AECOPD and hospitalization risk in patients with COPD but also impacts the long-term mortality risk on the patient. Lahousse et al followed up 2831 patients for 2 to 3 years and showed that frailty increased 4-fold risk for mortality in patients with COPD (HR $=4.03,95 \%$ CI: 1.22-13.30), ${ }^{5}$ which is consistent with our conclusion. In addition, our results suggested that although malnutrition was not the most common phenotypic characteristic of frailty (approximately 20.1\%), malnutrition increased the risk of mortality in patients with COPD by $115 \%$ ( $\mathrm{HR}=2.15$, 95\% CI: $1.02-4.51)$.

Frailty is an independent risk factor for exacerbations and progression of COPD, and patients with COPD are also more prone to frail. Although frail and COPD affect each other, both frailty and respiratory impairment can be modulated and treated. ${ }^{3,12,29}$ Rehabilitation is highly effective at strengthening skeletal muscles, improving symptom burden, physical function, and health status. ${ }^{30}$ Maddocks et al confirmed that frailty is an independent risk factor for lung rehabilitation noncompletion but appears to result in favorable rehabilitation 

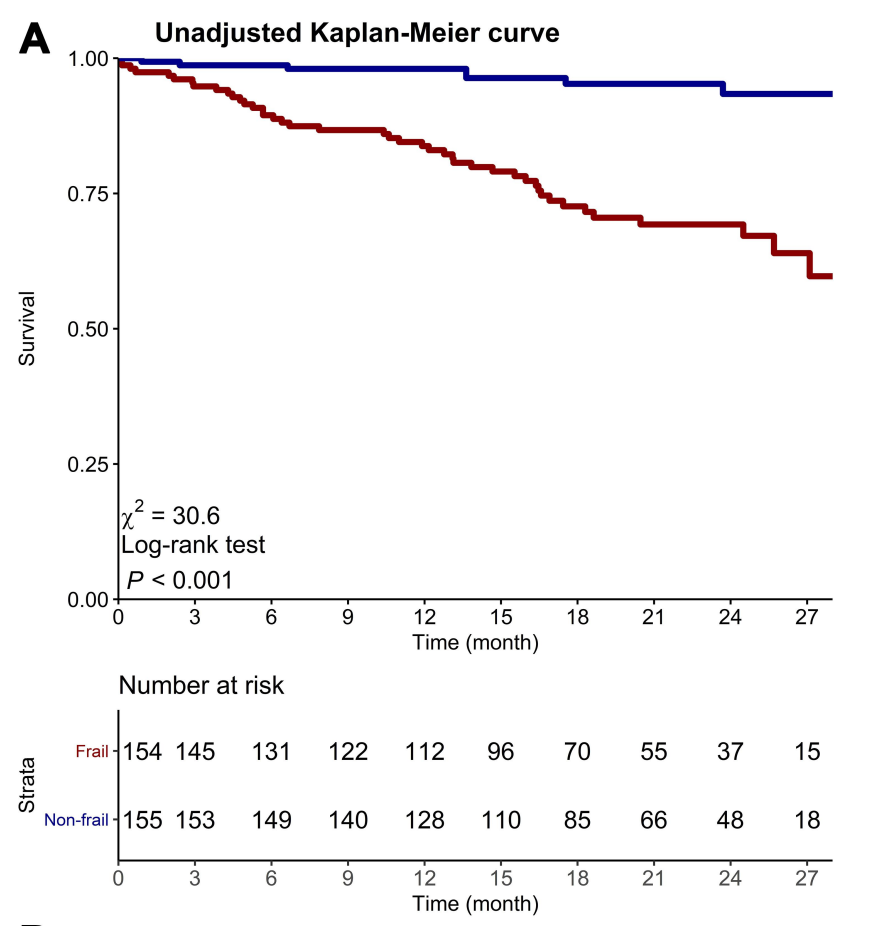

B cox model adjusted survival curve

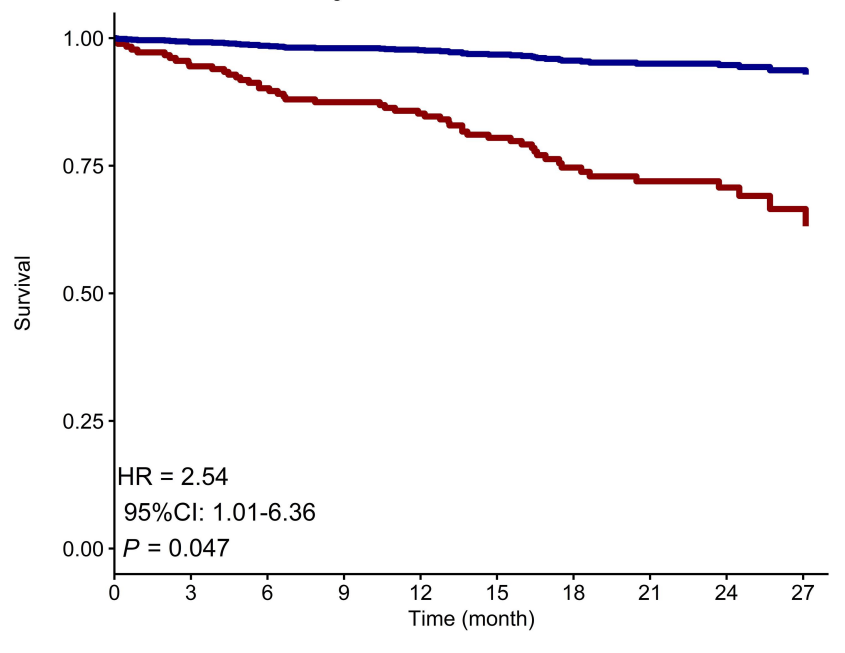

Strata - Frail - Non-frail

Figure 2 Comparison of unadjusted and adjusted survival curves of patients age $\geq$ 65 years with stable COPD stratified according to frailty (Fried phenotype score $\geq 3$ ). (A) Unadjusted Kaplan-Meier curve. (B) Survival curves adjusted by age, gender, $\mathrm{CCl}$, medication, GOLD severity, moderate-to-severe exacerbation history, and CAT. Frail patients had a higher mortality.

outcomes. ${ }^{8}$ The intervention of malnutrition requires targeted practice based on the clinical status of patients with COPD (stable or exacerbation), disease severity, and different activity levels. However, it also should pay attention to identify the specific goals of nutritional intervention (energy imbalance, sarcopenia, cachexia, frailty) in practice. ${ }^{31}$ Some researchers have suggested that medical nutrition therapy can provide sufficient levels of protein, the amino acid leucine, vitamin
$\mathrm{D}$, and polyunsaturated fatty acids, which may be beneficial for the treatment of sarcopenia and functional decline. ${ }^{32}$ Identification of frailty or pre-frailty in patients with COPD as early as possible and formulation and evaluation of tailored intervention programs may yield greater patient benefits. In practice, it is necessary to consider the efficacy and safety of therapy, establish a relationship of trust between physician and patient, create a common understanding of needs, clarify priorities, use personalized and multidisciplinary methods, provide flexible services, and offer information regarding riskbenefit decision-making. ${ }^{30}$

Recent studies have focused on describing groups of patients with clinically meaningful outcomes (symptoms, exacerbations, response to therapy, the rate of disease progression, or death) - COPD phenotypes, such as COPD-asthma overlap, emphysema-hyperinflation, and frequentexacerbation COPD phenotypes. ${ }^{17,29,33}$ Identification of these phenotypes is liable for clinical screening of unique high-risk populations and allows for tailored medical regimens. We believe that frailty phenotypes can similarly identify a unique population with a significantly increased risk of exacerbation, hospitalization, and death in patients with stable COPD. The high prevalence and poor prognosis of frail patients with COPD can serve as a warning that our traditional diseaseoriented medical model may be unable to meet the complex clinical needs of elderly patients with COPD, such as those with comorbidity and disability. An in-depth understanding of the relationship between frailty and COPD, early identification of pre-frail and frail patients with COPD, and timely intervention may provide a new perspective and clinical practice basis for improving the clinical prognosis of these patients and improving their quality of life.

\section{Limitations}

This study has some limitations. (1) This study was conducted in a single-center, with a small population and few end-point events, which reduces the generalizability of the findings. (2) Study participants included patients with stable COPD but not those with AECOPD, which limits the overall adaptability to the COPD population. (3) Physical activity determined for a frailty classification was self-reported. Failure to use electronic equipment to collect the physical activity of patients may be at risk of misclassification.

\section{Conclusion}

The results of this longitudinal cohort study indicated a high prevalence of frailty in elderly patients with stable COPD. Frailty significantly increased the incidence of acute 
exacerbation and hospitalization, as well as increased mortality in elderly patients with stable COPD.

\section{Abbreviations}

COPD, Chronic Obstructive Pulmonary Disease; AECOPD, acute exacerbations of COPD; IRR, incidence rate ratio; aHR, adjusted hazard ratios; CI, confidence intervals; FEV1, forced expiratory volume in 1 second; GOLD, Global Initiative for COPD; FVC, forced vital capacity; BMI, body mass index; CAT, COPD Assessment Test; mMRC, modified Medical Research Council; FFP, Fried frailty phenotype; CCI, Charlson comorbidity index; MNA-SF, Mini Nutritional AssessmentShort Form; ADL, Katz Activities of Daily Living; IADL, Lawton Instrumental Activities of Daily Living; SD, standard deviation; IQR, interquartile range; $\mathrm{BD}$, bronchodilator; ICS, inhaled corticosteroids; LABA, long-acting beta-agonists; LAMA, long-acting muscarinic antagonists.

\section{Data Sharing Statement}

Restrictions apply to the availability of data generated or analyzed during this study to preserve participant confidentiality. The corresponding author will on request detail the restrictions and any conditions under which access to some data may be provided.

\section{Ethics Approval and Informed Consent}

The research protocol of this study was approved by the Research Ethics Committee of Beijing Friendship Hospital and Capital Medical University (Beijing, China) and complied with the ethical guidelines of the Declaration of Helsinki (Project number: 2018-P2-137-01). All patients provided their signed informed consent before participating in this study.

\section{Acknowledgment}

We acknowledge the role of all patients, investigators, and support staff in performing the study.

\section{Author Contributions}

All authors contributed to data analysis, drafting or revising the article, have agreed on the journal to which the article will be submitted, gave final approval of the version to be published, and agree to be accountable for all aspects of the work.

\section{Funding}

This study was supported by the Beijing Municipal Administration of Hospitals Clinical Medicine
Development of Special Funding Support (Process No. ZYLX201838) and the Beijing Friendship Hospital of Capital Medical University Hospital Startup Funding (Process No. yyqdkt2017-37).

\section{Disclosure}

The authors report no conflicts of interest for this work.

\section{References}

1. Vogelmeier CF, Criner GJ, Martinez FJ, et al. Global Strategy for the Diagnosis, Management, and Prevention of Chronic Obstructive Lung Disease 2017 Report. GOLD Executive Summary. Am J Respir Crit Care Med. 2017;195(5):557-582. doi:10.1164/ rccm.201701-0218PP

2. Zhou M, Wang H, Zeng X, et al. Mortality, morbidity, and risk factors in China and its provinces, 1990-2017: a systematic analysis for the Global Burden of Disease Study 2017. Lancet. 2019;394 (10204):1145-1158. doi:10.1016/S0140-6736(19)30427-1

3. Dent E, Martin FC, Bergman H, et al. Management of frailty: opportunities, challenges, and future directions. Lancet. 2019;394 (10206):1376-1386. doi:10.1016/S0140-6736(19)31785-4

4. Fried LP, Tangen CM, Walston J, et al. Frailty in older adults: evidence for a phenotype. J Gerontol A Biol Sci Med Sci. 2001;56 (3):M146-M56. doi:10.1093/gerona/56.3.M146

5. Lahousse L, Ziere G, Verlinden VJA, et al. Risk of frailty in elderly with COPD: a population-based study. J Gerontol A Biol Sci Med Sci. 2016;71(5):689-695. doi:10.1093/gerona/glv154

6. Marengoni A, Vetrano DL, Manes-Gravina E, et al. The relationship between COPD and frailty: a Systematic Review and Meta-Analysis of Observational Studies. Chest. 2018;154(1):21-40. doi:10.1016/j. chest.2018.02.014

7. Vaz Fragoso CA, Enright PL, McAvay G, et al. Frailty and respiratory impairment in older persons. Am J Med. 2012;125(1):79-86. doi:10.1016/j.amjmed.2011.06.024

8. Maddocks M, Kon SSC, Canavan JL, et al. Physical frailty and pulmonary rehabilitation in COPD: a prospective cohort study. Thorax. 2016;71(11):988-995. doi:10.1136/thoraxjn1-2016-208460

9. Mittal N, Raj R, Islam EA, et al. The frequency of frailty in ambulatory patients with chronic lung diseases. J Prim Care Community Health. 2016;7(1):10-15. doi:10.1177/2150131915603202

10. Park SK, Richardson CR, Holleman RG, et al. Frailty in people with COPD, using the National Health and Nutrition Evaluation Survey dataset (2003-2006). Heart Lung. 2013;42(3):163-170. doi:10.1016/ j.hrtlng.2012.07.004

11. Mirza S, Benzo R. Chronic obstructive pulmonary disease phenotypes: implications for care. Mayo Clin Proc. 2017;92(7):1104-1112. doi:10.1016/j.mayocp.2017.03.020

12. Hoogendijk EO, Afilalo J, Ensrud KE, et al. Frailty: implications for clinical practice and public health. Lancet. 2019;394 (10206):1365-1375. doi:10.1016/S0140-6736(19)31786-6

13. Charlson ME, Pompei P, Ales KL, et al. A new method of classifying prognostic comorbidity in longitudinal studies: development and validation. J Chronic Dis. 1987;40(5):373-383. doi:10.1016/00219681(87)90171-8

14. Rubenstein LZ, Harker JO, Salvà $\mathrm{A}$, et al. Screening for undernutrition in geriatric practice: developing the short-form mini-nutritional assessment (MNA-SF). J Gerontol A Biol Sci Med Sci. 2001;56(6): M366-72. doi:10.1093/gerona/56.6.M366

15. Lawton MP, Brody EM. Assessment of older people: self-maintaining and instrumental activities of daily living. Gerontologist. 1969;9 (3):179-186. doi:10.1093/geront/9.3_Part_1.179 
16. Ehsani H, Mohler MJ, Golden T, et al. Upper-extremity function prospectively predicts adverse discharge and all-cause COPD readmissions: a pilot study. Int $J$ Chron Obstruct Pulmon Dis. 2019;14:39-49. doi:10.2147/COPD.S182802

17. Kennedy CC, Novotny PJ, LeBrasseur NK, et al. Frailty and clinical outcomes in chronic obstructive pulmonary disease. Ann Am Thorac Soc. 2019;16(2):217-224. doi:10.1513/AnnalsATS.201803-175OC

18. Davalos-Yerovi V, Marco E, Sánchez-Rodríguez D, et al. Sarcopenia according to the revised European Consensus on definition and diagnosis (EWGSOP2) criteria predicts hospitalizations and long-term mortality in rehabilitation patients with stable chronic obstructive pulmonary disease. J Am Med Dir Assoc. 2019;20(8):1047-1049. doi:10.1016/j.jamda.2019.03.019

19. Petermann-Rocha F, Chen MH, Gray SR, et al. New versus old guidelines for sarcopenia classification: what is the impact on prevalence and health outcomes? Age Ageing. 2020;49(2):300-304. doi:10.1093/ageing/afz126

20. Costanzo L, Vincentis AD, Iorio AD, et al. Impact of Low muscle mass and low muscle strength according to EWGSOP2 and EWGSOP1 in Community-Dwelling older people. $J$ Gerontol A Biol Sci Med Sci. 2020;75(7):1324-1330. doi:10.1093/gerona/ glaa063

21. Cruz-Jentoft AJ, Sayer AA. Sarcopenia. Lancet. 2019;393 (10191):2636-2646. doi:10.1016/S0140-6736(19)31138-9

22. Cruz-Jentoft AJ, Bahat G, Bauer J, et al. Sarcopenia: revised European consensus on definition and diagnosis. Age Ageing. 2019;48(1):16-31. doi:10.1093/ageing/afy169

23. van Helvoort HA, Heijdra YF, de Boer RC, et al. Six-minute walking-induced systemic inflammation and oxidative stress in muscle-wasted COPD patients. Chest. 2007;131(2):439-445. doi:10.1378/chest.06-1655

24. Bone AE, Hepgul N, Kon S, et al. Sarcopenia and frailty in chronic respiratory disease. Chron Respir Dis. 2017;14(1):85-99. doi:10.1177/1479972316679664
25. Shrikrishna D, Patel M, Tanner RJ, et al. Quadriceps wasting and physical inactivity in patients with COPD. Eur Respir J. 2012;40 (5):1115-1122. doi:10.1183/09031936.00170111

26. Jaitovich A, Barreiro E. Skeletal muscle dysfunction in chronic obstructive pulmonary disease. What we know and can do for our patients. Am J Respir Crit Care Med. 2018;198(2):175-186. doi:10.1164/rcem.201710-2140CI

27. Poberezhets V, Mostovoy Y, Demchuk H. Exacerbation of chronic obstructive pulmonary diseases as a risk factor of the skeletal muscle dysfunction. Lung India. 2019;36(3):188-192. doi:10.4103/lungindia. lungindia 18518

28. Bernabeu-Mora R, García-Guillamón G, Valera-Novella E, et al. Frailty is a predictive factor of readmission within 90 days of hospitalization for acute exacerbations of chronic obstructive pulmonary disease: a longitudinal study. Ther Adv Respir Dis. 2017;11 (10):383-392. doi:10.1177/1753465817726314

29. Han MK, Agusti A, Calverley PM, et al. Chronic obstructive pulmonary disease phenotypes: the future of COPD. Am J Respir Crit Care Med. 2010;182(5):598-604. doi:10.1164/rccm.200912-1843CC

30. Brighton LJ, Evans CJ, Man WDC, et al. Improving exercise-based interventions for people living with both COPD and frailty: a realist review. Int J Chron Obstruct Pulmon Dis. 2020;20(15):841-855. doi:10.2147/COPD.S238680

31. Collins PF, Yang IA, Chang YC, et al. Nutritional support in chronic obstructive pulmonary disease (COPD): an evidence update. $J$ Thorac Dis. 2019;11(Suppl 17):S2230-S7. doi:10.21037/ jtd.2019.10.41

32. Tessier AJ, Chevalier S. An update on protein, Leucine, Omega-3 fatty acids, and vitamin D in the prevention and treatment of sarcopenia and functional decline. Nutrients. 2018;10(8):1099. doi: 10.3390/nu10081099

33. Miravitlles M, Calle M, Soler-Cataluña JJ. Clinical phenotypes of COPD: identification, definition and implications for guidelines. Arch Bronconeumol. 2012;48:86-98. doi:10.1016/j.arbres.2011.10.007
Clinical Interventions in Aging

\section{Publish your work in this journal}

Clinical Interventions in Aging is an international, peer-reviewed journal focusing on evidence-based reports on the value or lack thereof of treatments intended to prevent or delay the onset of maladaptive correlates of aging in human beings. This journal is indexed on PubMed Central, MedLine, CAS, Scopus and the Elsevier
Bibliographic databases. The manuscript management system is completely online and includes a very quick and fair peer-review system, which is all easy to use. Visit http://www.dovepress.com/ testimonials.php to read real quotes from published authors. 